\title{
Superfluid behaviour of a two-dimensional
} Bose gas

\author{
Rémi Desbuquois, Lauriane Chomaz, Tarik Yefsah", Julian Léonard", Jérôme Beugnon, \\ Christof Weitenberg ${ }^{\star}$ and Jean Dalibard
}

Owing to thermal fluctuations, two-dimensional (2D) systems cannot undergo a conventional phase transition associated with the breaking of a continuous symmetry ${ }^{1}$. Nevertheless they may exhibit a phase transition to a state with quasi-longrange order via the Berezinskii-Kosterlitz-Thouless (BKT) mechanism². A paradigm example is the 2D Bose fluid, such as a liquid helium film ${ }^{3}$, which cannot condense at non-zero temperature although it becomes superfluid above a critical phase space density. The quasi-long-range coherence and the microscopic nature of the BKT transition were recently explored with ultracold atomic gases ${ }^{4-6}$. However, a direct observation of superfluidity in terms of frictionless flow is still missing for these systems. Here we probe the superfluidity of a 2D trapped Bose gas using a moving obstacle formed by a micrometre-sized laser beam. We find a dramatic variation of the response of the fluid, depending on its degree of degeneracy at the obstacle location.

'Flow without friction' is a hallmark of superfluidity ${ }^{7}$. It corresponds to a metastable state in which the fluid has a non-zero relative velocity $v$ with respect to an external body such as the wall of the container or an impurity. This metastable state is separated from the equilibrium state of the system $(v=0)$ by a large energy barrier, so that the flow can persist for a macroscopic time. The height of the barrier decreases as $v$ increases, and eventually passes below a threshold (proportional to the thermal energy) for a critical velocity $v_{\mathrm{c}}$. The microscopic mechanism limiting the barrier height depends on the nature of the defect and is associated with the creation of phonons and/or vortices ${ }^{7}$. Whereas the quantitative comparison between experiments and theory is complicated for liquid ${ }^{4} \mathrm{He}$, cold atomic gases in the weakly interacting regime are well suited for precise tests of many-body physics. In particular, superfluidity was observed in 3D atomic gases by stirring a laser beam or an optical lattice through bosonic ${ }^{8-12}$ or fermionic ${ }^{13}$ fluids and by observing the resulting heating or excitations. Here we transpose this search for dissipation-less motion to a disc-shaped, non-homogeneous 2D Bose gas. We use a small obstacle to locally perturb the system. The obstacle moves at constant velocity on a circle centred on the cloud, allowing us to probe the gas at a fixed density. We repeat the experiment for various atom numbers, temperatures and stirring radii and identify a critical point for superfluid behaviour.

Our experiments are performed with 2D Bose gases of $N=35,000-95,000{ }^{87} \mathrm{Rb}$ atoms confined in the vertical direction by the harmonic potential $W(z)$ and in the horizontal plane by the radially symmetric harmonic potential $V(r)$ (see ref. 14). The trap frequencies are $\omega_{r} / 2 \pi=25.0(5) \mathrm{Hz}$ in the horizontal a
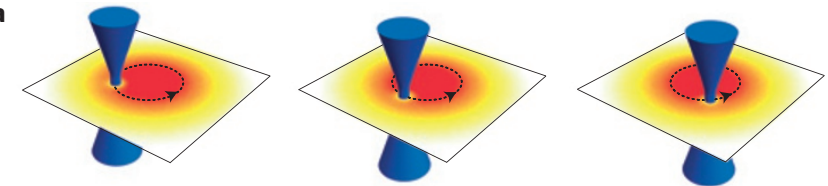

b

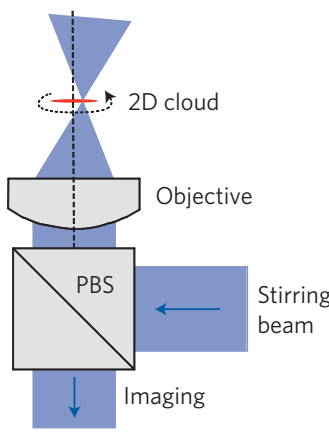

c

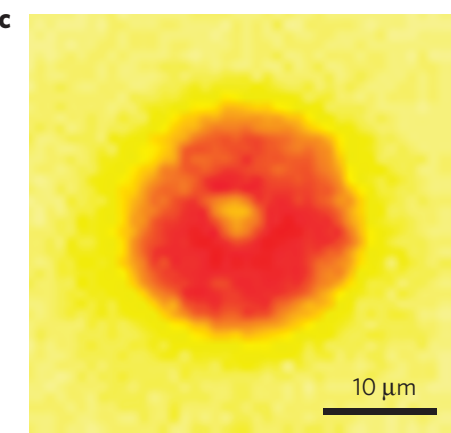

Figure 1 | Stirring a 2D Bose gas. a, A trapped $2 \mathrm{D}$ gas of ${ }^{87} \mathrm{Rb}$ atoms is perturbed by a focussed laser beam, which moves at constant velocity on a circle centred on the cloud. The stirring beam has a frequency greater than the ${ }^{87} \mathrm{Rb}$ resonance frequency ('blue detuning' of $\approx 2 \mathrm{~nm}$ ) and thus creates a repulsive potential which causes a dip in the density profile. $\mathbf{b}$, The stirring beam is focussed onto the 2D cloud via a microscope objective of numerical aperture 0.45 , which is also used for imaging. We overlap the two beam paths with a polarizing beam splitter (PBS) cube. The position of the stirring beam is controlled by a two-axis piezo-driven mirror. c, In situ false-colour image of the 2D cloud in the presence of the laser beam (average over six images). From the dip in the density we deduce the waist of the laser beam as $w_{0}=2.0(5) \mu \mathrm{m}$. In this image, the intensity of the beam is chosen three times higher than in the stirring experiment to make the hole clearly visible even in the centre of the cloud. We use similar images, but with the stirring beam switched off, to determine the temperature $T$ and the chemical potential $\mu$ from a fit of the Hartree-Fock prediction to the wings of the cloud ${ }^{14}$

plane and $\omega_{z} / 2 \pi=1.4(1) \mathrm{kHz}$ in the vertical direction. We use gases with temperature $T$ and central chemical potential $\mu$ in the range $65-120 \mathrm{nK}$ and $k_{\mathrm{B}} \times(35-60) \mathrm{nK}$, respectively, where $k_{\mathrm{B}}$ is the Boltzmann constant. The interaction energy per particle is given by $U_{\text {int }}=\left(\hbar^{2} \tilde{g} / m\right) n$, where $n$ is the $2 \mathrm{D}$ spatial density (typically 100 atoms $\mu \mathrm{m}^{-2}$ in the centre), $m$ the atomic mass, $\tilde{g}$ the dimensionless interaction strength and $\hbar$ is Planck's constant divided by $2 \pi$. Here $\tilde{g}=\sqrt{8 \pi} a / l_{z}=0.093$, where $a=5.3 \mathrm{~nm}$ 

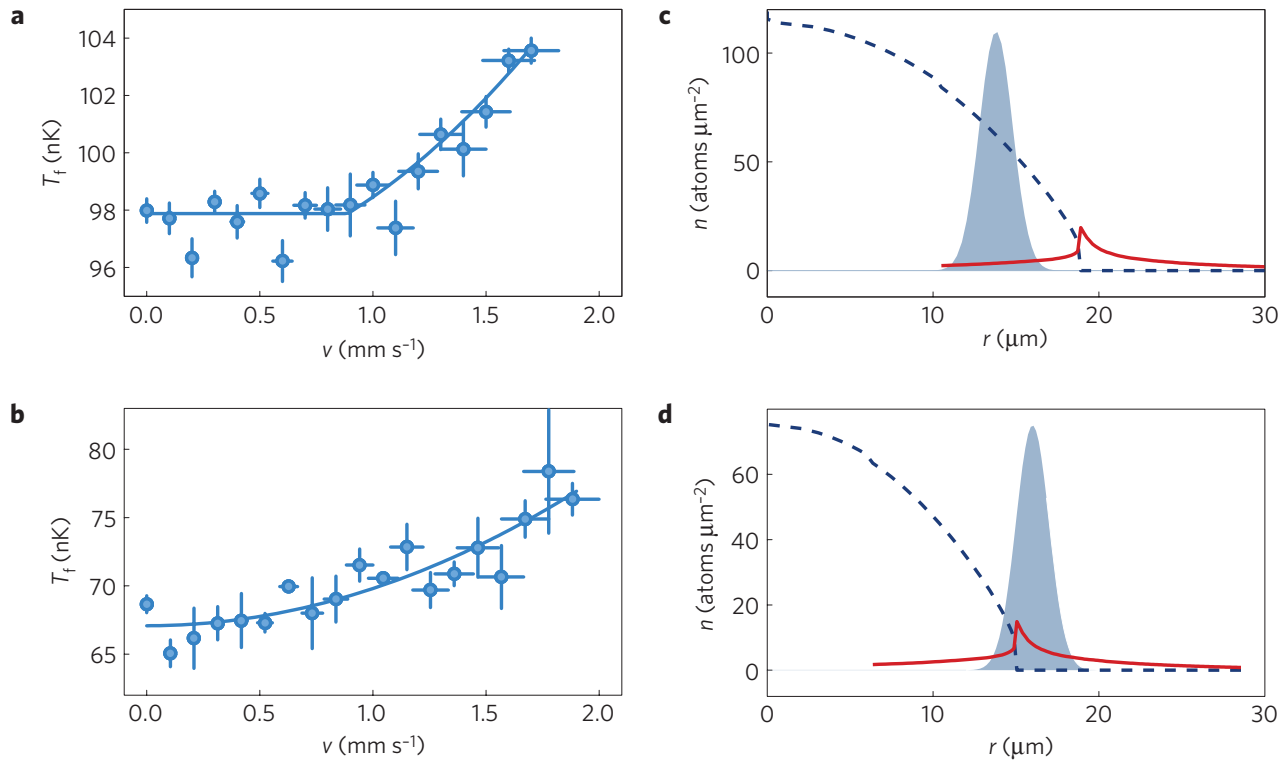

Figure 2 | Evidence for a critical velocity. Two typical curves of the temperature after stirring the laser beam at varying velocities. a, In the superfluid regime, we observe a critical velocity (here $v_{c}=0.87(9) \mathrm{mm} \mathrm{s}^{-1}$ ), below which there is no dissipation. $\mathbf{b}$, In the normal regime, the heating is quadratic in the velocity. The fitted heating coefficients are $\kappa=18(3) \mathrm{nK} \mathrm{s} \mathrm{mm}^{-2}$ and $\kappa=26(3) \mathrm{nK} \mathrm{s} \mathrm{mm} \mathrm{m}^{-2}$ in $\mathbf{a}$ and $\mathbf{b}$, respectively. The experimental parameters are $(N, \bar{T}, \mu, r)=\left(87,000,89 \mathrm{nK}, k_{\mathrm{B}} \times 59 \mathrm{nK}, 14.4 \mu \mathrm{m}\right)$ and $\left(38,000,67 \mathrm{nK}, \mathrm{k}_{\mathrm{B}} \times 39 \mathrm{nK}, 16.6 \mu \mathrm{m}\right)$ for $\mathbf{a}$ and $\mathbf{b}$, respectively, yielding $\mu_{\text {loc }} / \mathrm{k}_{\mathrm{B}} \bar{T}=0.36$ and $\mu_{\text {loc }} / k_{B} \bar{T}=0.04$. The data points are the average of typically ten shots. The $y$ error bars show the standard deviation. The $x$ error bars denote the spread of velocities along the size of the stirring beam ( $1 / \sqrt{e}$ radius). The solid line is a fit to the data according to equation (1). The stirring time is $0.2 \mathrm{~s}$ for all data points. Note that the three low-lying data points in a correspond to the completion of an odd number of half turns. For these data points, where we see a downshift of the temperature by approximately $1.5 \mathrm{nK}$, we also observe a displacement of the centre of mass of the cloud by a few micrometres.

c,d, Calculated radial density distribution for the clouds in $\mathbf{a}$ and $\mathbf{b}$, respectively. The dashed blue curve shows the superfluid density, the solid red curve shows the normal density. The stirring beam potential is indicated by the grey shaded area (in arbitrary units). The densities are calculated via the local density approximation from the prediction for an infinite uniform system ${ }^{16}$. The jump of the superfluid density from zero to a universal value of $4 / \lambda_{\mathrm{dB}}^{2}$ (where $\lambda_{\mathrm{dB}}$ is the thermal de Broglie wavelength) is a prominent feature of the BKT transition. The normal density makes a corresponding jump to keep the total density continuous.

is the $3 \mathrm{D}$ scattering length and $l_{z}=\sqrt{\hbar / m \omega_{z}}$. The energy $\hbar \omega_{z}$ $\left(k_{\mathrm{B}} \times 70 \mathrm{nK}\right)$ is comparable to $k_{\mathrm{B}} T$ and $U_{\text {int }}\left(\sim k_{\mathrm{B}} \times 40 \mathrm{nK}\right.$ at the trap centre). Thanks to Bose statistics, which limits to typically $10 \%$ the fractional atomic density in the axially excited states at the obstacle position, our gas is well described by the quasi-2D fluid model (see Supplementary material of ref. 14).

We stir the cloud with a laser beam which creates a repulsive potential with height $V_{\text {stir }} \approx k_{\mathrm{B}} \times 80 \mathrm{nK}$. This is at least twice the local chemical potential $\mu_{\mathrm{loc}}(r)=\mu-V(r)$. The beam has a Gaussian profile with a waist of $w_{0}=2.0(5) \mu \mathrm{m}$, which is larger than the local healing length $\xi=1 / \sqrt{\tilde{g} n}(\approx 0.3 \mu \mathrm{m}$ at the trap centre), but small compared to the size of the cloud (full width at half maximum $\approx 25 \mu \mathrm{m}$ ) (see Fig. 1 ). We stir for typically $t_{\text {stir }}=0.2 \mathrm{~s}$ at constant velocity $v$ in a circle of radius $r$ centred on the cloud. The intensity of the stirring beam is ramped on and off in $\approx 5 \mathrm{~ms}$ without any significant additional heating. Once the stirring beam is switched off, we let the cloud relax for $0.1 \mathrm{~s}$ and measure the temperature $T_{\mathrm{f}}$.

For each configuration $(N, T, r)$, we repeat this experiment for various $v$ from 0 to $2 \mathrm{~mm} \mathrm{~s}^{-1}$ and a fixed stirring time $t_{\text {stir. }}$. We find two different regimes for the response and we show an example of each in Fig. 2. In Fig. 2a, there is a clear threshold behaviour with no discernable dissipation below a critical velocity. In contrast, in Fig. 2b, the temperature increases without a threshold. We identify these behaviours as the superfluid and normal response, respectively. To model these data we choose for a given configuration the fit function

$$
T_{\mathrm{f}}(v)=T_{\mathrm{f}, 0}+\kappa \cdot t_{\mathrm{stir}} \cdot \max \left[\left(v^{2}-v_{\mathrm{c}}^{2}\right), 0\right]
$$

which describes the heating of a $2 \mathrm{D}$ superfluid in the presence of a moving point-like defect ${ }^{15}$. In equation (1) the three fit parameters are the temperature at zero velocity $T_{\mathrm{f}, 0}$, the heating coefficient $\kappa$, and the critical velocity $v_{\mathrm{c}}$. In the normal state, the fit gives $v_{\mathrm{c}} \sim 0$ and the according quadratic heating stems from the linear scaling of the drag force. In the absence of the stirring beam, there is no significant heating and we measure the temperature $T_{\mathrm{i}}$. The presence of the stirring beam at zero velocity leads to a 'background heating' $T_{\mathrm{f}, 0}-T_{\mathrm{i}} \sim 10 \mathrm{nK}$, which we attribute to photon scattering. In the following, we use the mean temperature $\bar{T}=\left(T_{\mathrm{i}}+T_{\mathrm{f}, 0}\right) / 2$ to characterize the cloud.

In Fig. 3, we summarize our data obtained for different configurations $(N, \bar{T}, r)$. We show in Fig. 3 a the fitted critical velocities versus the single parameter $\mu_{\mathrm{loc}}(r) / k_{\mathrm{B}} \bar{T}$. The relevance of this parameter results from two points. First, because of the local character of the excitation, the response of the fluid to the moving perturbation is expected to be similar to that of a uniform gas with the same temperature and the chemical potential $\mu_{\text {loc }}$. Second, the scale invariance of the weakly-interacting 2D Bose gas implies that the thermodynamic properties do not depend separately on $\mu$ and $T$, but only on the ratio $\mu / k_{\mathrm{B}} T$ (see refs $14,16,17$ ). In particular, this ratio is univocally related to the phase space density, and thus characterizes the degree of degeneracy of the cloud.

Remarkably, the ensemble of our data for $v_{\mathrm{c}}$ when plotted as a function of $\mu_{\text {loc }} / k_{\mathrm{B}} \bar{T}$ shows a threshold between values compatible with zero and clearly non-zero values. This threshold is located at $\mu_{\text {loc }} / k_{\mathrm{B}} \bar{T} \approx 0.24$, above the prediction $\left(\mu / k_{\mathrm{B}} T\right)_{\mathrm{c}}=0.15$ for the superfluid phase transition in a uniform system ${ }^{16}$ with $\tilde{g}=0.093$. If we assume that the stirrer must stand entirely in the superfluid core to yield a non-zero critical velocity, then the deviation can be 
a

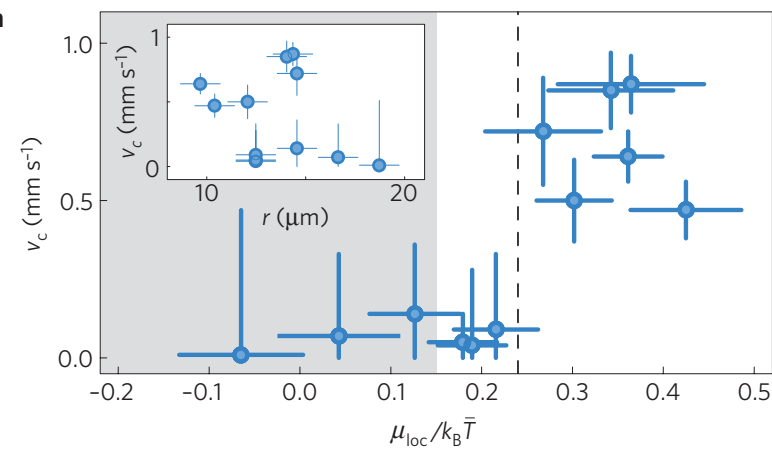

b

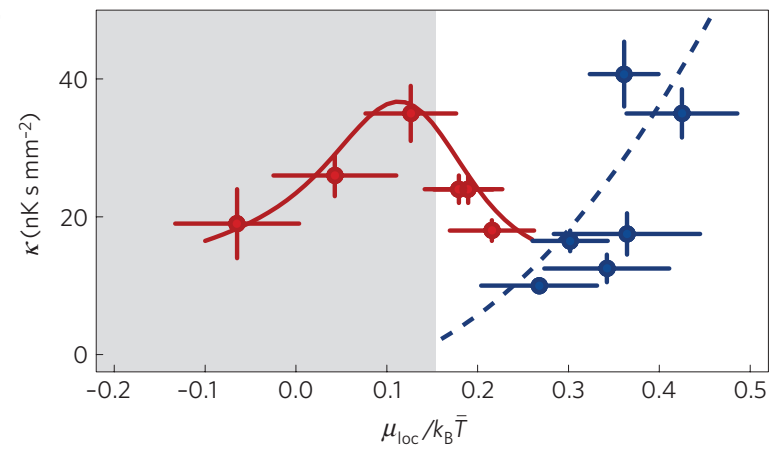

Figure 3 | Superfluid behaviour across the BKT transition. a, The critical velocities $v_{\mathrm{C}}$ obtained from the curves as in Fig. 2 plotted versus the single parameter $\mu_{\text {loc }} / k_{\mathrm{B}} \bar{T}$, which is the relevant quantity due to the scale invariance of the weakly-interacting 2D Bose gas. Our data show a threshold between critical velocities compatible with zero and clearly non-zero critical velocities. It is located at $\mu_{\text {loc }} / k_{\mathrm{B}} \bar{T} \approx 0.24$ (dashed line), somewhat above the prediction $\left(\mu_{\mathrm{loc}} / k_{\mathrm{B}} T\right)_{\mathrm{C}}=0.15$ for the BKT transition in an infinite uniform system ${ }^{16}$ (the grey shaded area indicates the normal state by this prediction). The inset to a shows the critical velocity plotted versus the stirring radius $r$. Owing to the different atom numbers and temperatures of the clouds, we can find superfluid or normal behaviour for the same radius. $\mathbf{b}$, The heating coefficient $\kappa$ as a function of $\mu_{\text {loc }} / k_{\mathrm{B}} \bar{T}$ for the normal data (red circles) and the superfluid data (blue circles). The red solid line shows a fit of $\kappa$ linear in the normal density, as expected from a single-particle model. The blue dashed line shows an empirical fit quadratic in the superfluid density. The calculation for the densities assumes $\bar{T}=90 \mathrm{nK}$ and the densities are averaged over the size of the stirring beam. The $x$ error bars indicate the region of $\mu_{\mathrm{loc}} / \mathrm{k}_{\mathrm{B}} \bar{T}$ that is traced by the stirring beam due to its size (using the $1 / \sqrt{e}$ width of the beam) and due to the 'background heating'. The $y$ error bars are fitting errors.

attributed to the non-zero width of the stirring beam. The range of $\mu_{\mathrm{loc}} / k_{\mathrm{B}} \bar{T}$ corresponding to the extent of this beam is indicated by the horizontal error bars in Fig. 3a. Note that the finite size of our trapped atomic clouds might also shift the BKT transition, but the effect is expected to be small (a few per cent) and in the opposite direction ${ }^{16}$.

We limit the presented stirring radii to $r \geq 10 \mu \mathrm{m}$ such that the stirring frequencies $\omega=v / r$ for the relevant velocities $v \sim v_{\mathrm{c}}$ are well below $\omega_{r}$. Indeed, smaller radii correspond to a larger centripetal acceleration. This could lead to additional heating via the phonon analog of synchrotron radiation, as observed in the formally similar context of capillary waves generated by a rotating object ${ }^{18}$.

For a homogeneous system, the value of the critical velocity is limited by two dissipation mechanisms, the excitation of phonons or vortices. For a point-like obstacle ${ }^{15}$, phonon excitation dominates and $v_{\mathrm{c}}$ is equal to the speed of sound, given in the zero-temperature limit by $c_{\mathrm{s}}=\hbar \sqrt{\tilde{g} n} / m\left(\approx 1.6 \mathrm{~mm} \mathrm{~s}^{-1}\right.$ for $n=50$ atoms $\mu \mathrm{m}^{-2}$ ) (this situation is described by the celebrated Landau criterion $\left.{ }^{7}\right)$. When the obstacle size $w_{0}$ increases and becomes comparable to $\xi$, dissipation via the nucleation of vortexantivortex pairs (vortex rings in 3D) becomes significant ${ }^{19-21}$. The corresponding $v_{\mathrm{c}}$ is then notably reduced with respect to $c_{\mathrm{s}}$. In the limit of very large obstacles $\left(w_{0} \gg \xi\right)$, an analytical analysis of the superfluid flow stability yields $v_{\mathrm{c}} \sim \hbar / m w_{0} \ll c_{\mathrm{s}}$ (see refs 22,23). With an obstacle size $w_{0} \gtrsim \xi$, our experimental situation is intermediate between these two asymptotic regimes. For a non-homogeneous system such as ours with the stirring obstacle close to the border of the expected superfluid regime, one can also excite surface modes ${ }^{24,25}$, which constitute a further dissipation mechanism.

Our measured critical velocities are in the range $0.5-1.0 \mathrm{~mm} \mathrm{~s}^{-1}$, that is, $v_{\mathrm{c}} / c_{\mathrm{s}}=0.3-0.6$. By contrast, previous experiments in $3 \mathrm{D}$ clouds found lower fractions, $v_{\mathrm{c}} / c_{\mathrm{s}} \sim 0.1$ (see ref. 9). The difference may be due to the larger size of the obstacles that were used, and to the average along the axis of the stirring beam of the density distribution in the $3 \mathrm{D}$ gas $^{26}$. The dominant dissipation mechanism could be revealed, for example, by directly observing the created vortex pairs as in ref. 12 or interferometrically detecting the Cerenkov-like wave pattern for $v>c_{\mathrm{s}}$ as in experiments with a nonequilibrium $2 \mathrm{D}$ superfluid of exciton-polariton quasi-particles ${ }^{27}$.

Figure $3 \mathrm{~b}$ shows the fitted heating coefficients $\kappa$ for the normal (red circles) and superfluid data (blue circles). In the normal region, we expect the heating to scale linearly with the normal density $n_{\text {no }}$ (see ref. 10). Using the prediction of ref. 16 for $\bar{n}_{\text {no }}$ (averaged over the size of the stirring beam) we fit $\kappa=a_{1} \cdot \bar{n}_{\text {no }}$ and obtain $a_{1} \approx 3 \times 10^{-6} \mathrm{nK}$ s. This value is in reasonable agreement with the prediction of a model ${ }^{10}$ of a single particle with a thermal velocity distribution of mean $\bar{v}=\sqrt{\pi k_{\mathrm{B}} T / 2 m}$ colliding with a moving hard wall of width $L=w_{0}$, yielding $a_{1}=16 \mathrm{~mL} \bar{v} / \pi N k_{\mathrm{B}} \sim 6 \times 10^{-6} \mathrm{nK} \mathrm{s}$ (for $N=65,000$ and $T=90 \mathrm{nK}$ ). In particular our data nicely reproduce the maximum of $\bar{n}_{\text {no }}$ around the expected superfluid transition point. In the superfluid case and $v>v_{c}$, we empirically fit a quadratic scaling of the heating with density $\kappa=a_{2} \cdot n_{\mathrm{SF}}^{2}$ and find $a_{2}=8 \times 10^{-9} \mathrm{nK} \mathrm{s} \mu \mathrm{m}^{2}$. In principle, one could develop a more refined model to describe the superfluid region, by taking into account the coexistence of the normal and superfluid states via the sum of two heating terms. However, within the accuracy of our data, we did not find any evidence for the need of such a more refined description.

We have presented a direct proof of the superfluid character of a trapped 2D Bose gas. An interesting extension of our work would be the study of superfluidity from the complementary point of view of persistent currents, by adapting to $2 \mathrm{D}$ the pioneering experiments performed in 3D toroidal traps ${ }^{28-30}$.

\section{Received 21 May 2012; accepted 25 June 2012; published online 29 July 2012}

\section{References}

1. Mermin, N. D. \& Wagner, H. Absence of ferromagnetism or antiferromagnetism in one- or two-dimensional isotropic Heisenberg models. Phys. Rev. Lett. 17, 1133-1136 (1966).

2. Minnhagen, P. The two-dimensional Coulomb gas, vortex unbinding, and superfluid-superconducting films. Rev. Mod. Phys. 59, 1001-1066 (1987).

3. Bishop, D. J. \& Reppy, J. D. Study of the superfluid transition in two-dimensional ${ }^{4}$ He films. Phys. Rev. Lett. 40, 1727-1730 (1978).

4. Hadzibabic, Z., Krüger, P., Cheneau, M., Battelier, B. \& Dalibard, J. Berezinskii-Kosterlitz-Thouless crossover in a trapped atomic gas. Nature 441, 1118-1121 (2006).

5. Cladé, P., Ryu, C., Ramanathan, A., Helmerson, K. \& Phillips, W. D. Observation of a 2D Bose gas: From thermal to quasicondensate to superfluid. Phys. Rev. Lett. 102, 170401 (2009).

6. Tung, S., Lamporesi, G., Lobser, D., Xia, L. \& Cornell, E. A. Observation of the presuperfluid regime in a two-dimensional Bose gas. Phys. Rev. Lett. 105, 230408 (2010).

7. Leggett, A. J. Quantum Liquids: Bose Condensation and Cooper Pairing in Condensed-Matter Systems (Oxford Univ. Press, 2006).

8. Raman, C. et al. Evidence for a critical velocity in a Bose-Einstein condensed gas. Phys. Rev. Lett. 83, 2502-2505 (1999). 
9. Onofrio, R. et al. Observation of superfluid flow in a Bose-Einstein condensed gas. Phys. Rev. Lett. 85, 2228-2231 (2000).

10. Raman, C., Onofrio, R., Vogels, J. M., Abo-Shaeer, J. R. \& Ketterle, W. Dissipationless flow and superfluidity in gaseous Bose-Einstein condensates. J. Low Temp. Phys. 122, 99-116 (2001).

11. Engels, P. \& Atherton, C. Stationary and nonstationary fluid flow of a Bose-Einstein condensate through a penetrable barrier. Phys. Rev. Lett. 99, 160405 (2007)

12. Neely, T. W., Samson, E. C., Bradley, A. S., Davis, M. J. \& Anderson, B. P. Observation of vortex dipoles in an oblate Bose-Einstein condensate. Phys. Rev. Lett. 104, 160401 (2010).

13. Miller, D. E. et al. Critical velocity for superfluid flow across the BEC-BCS crossover. Phys. Rev. Lett. 99, 070402 (2007).

14. Yefsah, T., Desbuquois, R., Chomaz, L., Günter, K. J. \& Dalibard, J. Exploring the thermodynamics of a two-dimensional Bose gas. Phys. Rev. Lett. 107, 130401 (2011).

15. Astrakharchik, G. E. \& Pitaevskii, L. P. Motion of a heavy impurity through a Bose-Einstein condensate. Phys. Rev. A 70, 013608 (2004).

16. Prokof ev, N. \& Svistunov, B. Two-dimensional weakly interacting Bose gas in the fluctuation region. Phys. Rev. A 66, 043608 (2002).

17. Hung, C-L., Zhang, X., Gemelke, N. \& Chin, C. Observation of scale invariance and universality in two-dimensional Bose gases. Nature 470, 236-239 (2011).

18. Chepelianskii, A. D., Chevy, F. \& Raphaël, E. Capillary-gravity waves generated by a slow moving object. Phys. Rev. Lett. 100, 074504 (2008).

19. Langer, J. S. \& Fisher, M. E. Intrinsic critical velocity of a superfluid. Phys. Rev. Lett. 19, 560-563 (1967).

20. Frisch, T., Pomeau, Y. \& Rica, S. Transition to dissipation in a model of superflow. Phys. Rev. Lett. 69, 1644-1647 (1992).

21. Winiecki, T., McCann, J. F. \& Adams, C. S. Pressure drag in linear and nonlinear quantum fluids. Phys. Rev. Lett. 82, 5186-5189 (1999).

22. Stießberger, J. S. \& Zwerger, W. Critical velocity of superfluid flow past large obstacles in Bose-Einstein condensates. Phys. Rev. A 62, 061601(R) (2000).

23. Crescimanno, M., Koay, C. G., Peterson, R. \& Walsworth, R. Analytical estimate of the critical velocity for vortex pair creation in trapped Bose condensates. Phys. Rev. A 62, 063612 (2000).
24. Dalfovo, F., Giorgini, S., Guilleumas, M., Pitaevskii, L. \& Stringari, S Collective and single-particle excitations of a trapped Bose gas. Phys. Rev. A 56, 3840-3845 (1997).

25. Dubessy, R., Liennard, T., Pedri, P. \& Perrin, H. Critical rotation of an annular superfluid Bose gas. PRA (in the press); preprint at http://arxiv.org/abs/1204.6183v1 (2012).

26. Fedichev, P. O. \& Shlyapnikov, G. V. Critical velocity in cylindrical Bose-Einstein condensates. Phys. Rev. A 63, 045601 (2001).

27. Amo, A. et al. Superfluidity of polaritons in semiconductor microcavities. Nature Phys. 5, 805-810 (2009).

28. Ryu, C. et al. Observation of persistent flow of a Bose-Einstein condensate in a toroidal trap. Phys. Rev. Lett. 99, 260401 (2007).

29. Ramanathan, A. et al. Superflow in a toroidal Bose-Einstein condensate: An atom circuit with a tunable weak link. Phys. Rev. Lett. 106, 130401 (2011).

30. Moulder, S., Beattie, S., Smith, R. P., Tammuz, N. \& Hadzibabic, Z. Quantised superflow glitches in an annular Bose-Einstein condensate. Preprint at http://arxiv.org/abs/1112.0334v2 (2012)

\section{Acknowledgements}

We would like to thank I. Carusotto, M. Holzmann, S. Nascimbéne, H. Perrin, L. Pitaevskii and W. Zwerger for helpful discussions. We acknowledge funding by IFRAF, ANR (project BOFL), the Alexander von Humboldt foundation (C.W.) and DGA (L.C.) Laboratoire Kastler Brossel is a research unit of École normale supérieure and Université Pierre et Marie Curie, associated with CNRS.

\section{Author contributions}

The experiments were carried out by R.D., L.C., T.Y. and C.W.; the stirring laser set-up was developed by J.L. and J.B.; C.W., R.D. and L.C. analysed the data; C.W. wrote the manuscript with the input from all coauthors; J.B. and J.D. planned and supervised the project.

\section{Additional information}

Reprints and permissions information is available online at www.nature.com/reprints. Correspondence and requests for materials should be addressed to C.W.

\section{Competing financial interests}

The authors declare no competing financial interests. 